EPJ Web of Conferences $\mathrm{T}, 05014$ (2010)

DOI:10.1051/epjconf/20100105014

(C) Owned by the authors, published by EDP Sciences, 2010

\title{
The proton-proton scattering without Coulomb force renormalization
}

\author{
R. Skibiński ${ }^{1, a}$, J.Golak ${ }^{1}$, H.Witała ${ }^{1}$, and W.Glöckle ${ }^{2}$ \\ 1 M. Smoluchowski Institute of Physics, Jagiellonian University,PL-30059 Kraków, Poland \\ 2 Institut für Theoretische Physik II, Ruhr-Universität Bochum, D-44780 Bochum, Germany
}

\begin{abstract}
We demonstrate numerically that proton-proton (pp) scattering observables can be determined directly by standard short range methods using a screened pp Coulomb force without renormalization. We numerically investigate solutions of the 3-dimensional Lippmann-Schwinger (LS) equation for an exponentially screened Coulomb potential. For the limit of large screening radii we confirm analytically predicted properties for off-shell, half-shell and on-shell elements of the Coulomb t-matrix.
\end{abstract}

\section{Introduction}

Recently [1], we proposed a method to obtain pp scattering observables using a screened Coulomb force in the standard framework of short range interactions. Namely, despite the fact that the screening limit of the on-shell scattering amplitude does not exist and acquires an oscillating phase factor if the screening radius goes to infinity [2-5], it is still possible to obtain pp observables without renormalization of the scattering amplitude.

The two-body screened Coulomb t-matrix is an input to study proton-deuteron processes. In a series of papers [6, 7] related to the pd system, the screened Coulomb t-matrix was used in two forms: in the partial wave decomposition and in the direct 3-dimensional form $<\mathbf{p}^{\prime}\left|t_{c}^{R}(E)\right| \mathbf{p}>$. The latter one is a solution of the 3-dimensional two-body LS equation driven by the screened Coulomb potential. Using this form one takes into account the full action of the Coulomb force in the pd system. This allows to avoid big number of partial waves required otherwise.

The off-the-energy-shell, half-shell and on-shell properties of the screened Coulomb t-matrix have been studied analytically in the past [2-4,8-10]. These investigations, however, mostly rely on insight gained for fixed number of partial wave states. Their mathematical rigour in the summation of the partial wave sum to infinity leaves room for improvement. Therefore, we study numerically the screening limit of $<\mathbf{p}^{\prime}\left|t_{c}^{R}(E)\right| \mathbf{p}>$ for the off-, half- and onshell matrix elements and compare obtained results with the unscreened pure Coulomb force predictions. For the half-the-energy-shell and for the on-the-energy-shell elements we also checked the scale of effects of the renormalization procedure. This allows to point the values of the screening range for which the screening limit is reached with the adequate accuracy.

This paper is organized as follows: in Section II we discuss pp scattering observables. In Section III the 3-dimen-

a e-mail: roman.skibinski@uj.edu.pl sional LS equation for the 3-dimensional, screened Coulomb $t$-matrix is presented and examples of its matrix elements and their properties are shown in Section IV. We summarize in Section V.

\section{The on-shell pp t-matrix with screened Coulomb potential and the pp observables}

Let $V_{c}^{R}$ be the screened Coulomb potential between 2 protons, which turns into the pure pp Coulomb potential for $R$, the screening radius, going to infinity. Together with the strong interaction $\mathrm{V}$ this determines the 2-body $\mathrm{pp}$ t-matrix via the LS equation

$$
t=V+V_{c}^{R}+\left(V+V_{c}^{R}\right) G_{0} t,
$$

where $G_{0}$ is the free propagator. That equation is solved at the $p p$ c.m. energy $E=\frac{p^{2}}{m_{p}}$ projected on a set of partial wave basis states $\mid p(l s) j m ; t m_{t}>$, with $p, l, s, j$ and $m$ being the relative momentum, orbital angular momentum, total spin, total angular momentum and its magnetic quantum number.

The total isospin quantum numbers for two protons are $t=1$ and $m_{t}=-1$. This leads to the on-the-energy-shell t-matrix element

$$
<p\left(l^{\prime} s^{\prime}\right) j^{\prime} m^{\prime}|t| p(l s) j m>=\delta_{s^{\prime} s} \delta_{j^{\prime} j} \delta_{m^{\prime} m} t_{l^{\prime} l}^{s j}(p, p),
$$

where the Pauli principle dictates $(-)^{l+s}=1$ and we assumed $s$ to be conserved.

The full 3-dimensional antisymmetrized on-shell t-matrix is given as

$$
<\mathbf{p}^{\prime} m_{1}^{\prime} m_{2}^{\prime}\left|t\left(1-P_{12}\right)\right| \mathbf{p} m_{1} m_{2}>,
$$

where $m_{i}\left(m_{i}^{\prime}\right)$ are the individual spin magnetic quantum numbers and $\mathbf{p}=p \hat{p}, \mathbf{p}^{\prime}=p \hat{p}^{\prime}$ the initial and final relative momenta. 
The standard partial wave decomposition leads to

$$
\begin{aligned}
& <\mathbf{p}^{\prime} m_{1}^{\prime} m_{2}^{\prime}\left|t\left(1-P_{12}\right)\right| \mathbf{p} m_{1} m_{2}>=\sum_{s}\left(\frac{1}{2} \frac{1}{2} s, m_{1}^{\prime} m_{2}^{\prime} m_{s}^{\prime}\right) \\
& \left(\frac{1}{2} \frac{1}{2} s, m_{1} m_{2} m_{s}\right) \sum_{j=0}^{\infty} \sum_{m=-j}^{j} \sum_{l^{\prime}=|j-s|}^{j+s} \sum_{l=|j-s|}^{j+s} \sum_{m_{l}^{\prime}} \\
& \left(l^{\prime} s j, m_{l}^{\prime}, m_{s}^{\prime}, m\right) Y_{l^{\prime} m_{l}^{\prime}}\left(\hat{p}^{\prime}\right) t_{l^{\prime} l}^{s j}(p, p)\left(1+(-)^{l+s}\right) \\
& \sum_{m_{l}}\left(l s j, m_{l} m_{s}, m\right) Y_{l m_{l}}^{*}(\hat{p}) .
\end{aligned}
$$

The strong force can be neglected beyond a certain $j_{\max }$ and there only the screened Coulomb t-matrix $t_{c l}^{R}$ is present, which is diagonal in $l$ and independent of $s$ and $j$. Next, one adds and subtracts a finite sum up to $j_{\text {max }}$ with $t_{c l}^{R}$ only and this completes the infinite sum over $j$ containing only $t_{c l}^{R}$. That infinite sum is identical to the 3-dimensional antisymmetric screened Coulomb t-matrix. Thus (4) turns into

$$
\begin{aligned}
< & \mathbf{p}^{\prime} m_{1}^{\prime} m_{2}^{\prime}\left|t\left(1-P_{12}\right)\right| \mathbf{p} m_{1} m_{2}>=\delta_{m_{1}^{\prime} m_{1}} \delta_{m_{2}^{\prime} m_{2}}<\mathbf{p}^{\prime}\left|t_{c}^{R}\right| \mathbf{p}> \\
- & \delta_{m_{1}^{\prime} m_{2}} \delta_{m_{2}^{\prime} m_{1}}<\mathbf{p}^{\prime}\left|t_{c}^{R}\right|-\mathbf{p}>+\sum_{s}\left(\frac{1}{2} \frac{1}{2} s, m_{1}^{\prime} m_{2}^{\prime} m_{s}^{\prime}\right) \\
& \left(\frac{1}{2} \frac{1}{2} s, m_{1} m_{2} m_{s}\right) \sum_{j=0}^{\infty} \sum_{m=-j}^{j} \sum_{l^{\prime}=|j-s|}^{j+s} \sum_{l=|j-s|}^{j+s} \sum_{m_{l}^{\prime}} \\
& \left(l^{\prime} s j, m_{l}^{\prime}, m_{s}^{\prime}, m\right) Y_{l^{\prime} m_{l}^{\prime}}\left(\hat{p}^{\prime}\right)\left(t_{l^{\prime} l}^{s j}(p, p)-\delta_{l^{\prime}} t_{c l}^{R}\right)\left(1+(-)^{l+s}\right) \\
& \sum_{m_{l}}\left(l s j, m_{l} m_{s}, m\right) Y_{l m_{l}}^{*}(\hat{p}) .
\end{aligned}
$$

The limit of that expression does not exist for $R \rightarrow$ $\infty[3,4]$. Then each term in (5) acquires the same infinitely oscillating phase factor $e^{2 i \Phi_{R}(p)}$, where $\Phi_{R}(p)$ depends on the type of the screening used. If one is interested in phase shifts it is unavoidable to keep track of this oscillating factor which in that context runs under the name renormalization [5]. However, if one is interested in the pp observables, the cross section and spin observables, where the on-shell t-matrix appears together with its complex conjugate, the oscillating factor drops out. Note that $\Phi_{R}(p)$ is independent of spin magnetic quantum numbers.

Thus, the pp observables based on the strong and the screened Coulomb force can be calculated without renormalization using standard short range methods. Only the values of the parameter $R$ at which the observables get independent of $R$ have to be established.

In this work we use the exponential type of screening which depends on two parameters, the screening radius $R$ and the power $n$ :

$$
V_{c}^{R}(r)=\frac{e^{2}}{r} e^{-\left(\frac{r}{R}\right)^{n}}
$$

At a given value $n$, which we take from $n=1$ up to 4 , the pure Coulomb potential is restored for $R \rightarrow \infty$. The phase factor for the exponential screening reads [11]

$$
\Phi_{R}(p)=-\eta[\ln (2 p R)-\gamma / n]
$$

where $\gamma=0.5772 \ldots$ is the Euler number and $\eta=\frac{m_{p} e^{2}}{2 p}$ the Sommerfeld parameter.

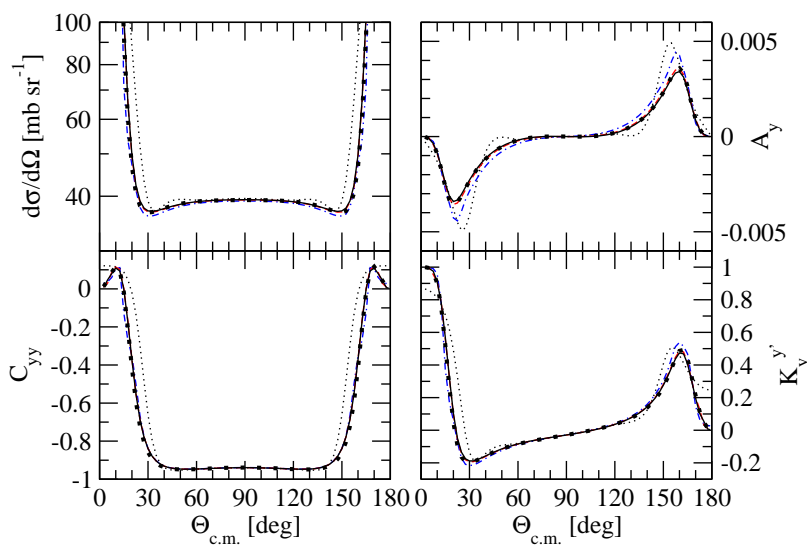

Fig. 1. (color online) The pp scattering cross section $\left(\frac{d \sigma}{d \Omega}\right)$, analyzing power $\left(A_{y}\right)$, spin correlation coefficient $\left(C_{y y}\right)$, and spin transfer coefficient $\left(K_{y}^{y^{\prime}}\right)$ at $E_{p}^{l a b}=13 \mathrm{MeV}$ as a function of the c.m. scattering angle calculated with the screened Coulomb force and the CD Bonn nucleon-nucleon potential [13], which is kept for the partial waves up to $j \leq 3$. The screened results are for $n=4$ and different values of the screening radius $R: R=20 \mathrm{fm}$ (dotted line), $R=60 \mathrm{fm}$ (dashed-dotted line), $R=120 \mathrm{fm}$ (dashed line), and $R=180 \mathrm{fm}$ (solid line). The thick dots are the VincentPhatak's exact results.

In Fig. 1 we show for several pp observables the convergence with respect to $R$ for $n=4$ at $E_{p}^{l a b}=13 \mathrm{MeV}$. The resulting limiting values agree very well with the exact standard predictions obtained using the Vincent-Phatak method [12]. For this energy the screening limit for observables is achieved at $R=120 \mathrm{fm}$.

In Fig. 2 we demonstrate for the same observables the independence on the value of $n$ at sufficiently large R-value. Again the agreement with the results of the Vincent-Phatak method is seen. The deviation with respect to the latter predictions and values of $n=1,2,3$ and 4 and screening radius $R=120 \mathrm{fm}$ is below $1 \%$.

\section{The 3-dimensional Lippmann-Schwinger equation}

Let us regard the LS equation for two protons interacting only with the screened Coulomb potential $V_{c}^{R}$. The t-matrix element $<\mathbf{p}^{\prime}\left|t_{c}^{R}\left(E=\frac{k^{2}}{m_{p}}\right)\right| \mathbf{p}>\equiv t_{c}^{R}\left(p^{\prime}, p, x=\hat{p} \cdot \hat{p}^{\prime} ; E\right)$ fulfills for given energy $E$ the equation [14]

$$
\begin{aligned}
& t_{c}^{R}\left(p^{\prime}, p, x\right)=\frac{1}{2 \pi} v_{c}^{R}\left(p^{\prime}, p, x, 1\right)+\int_{0}^{\infty} d p " p, 2 \\
& \int_{-1}^{1} d x " v_{c}^{R}\left(p^{\prime}, p ", x, x^{\prime \prime}\right) \frac{1}{E+i \epsilon-\frac{p^{\prime \prime}}{m_{p}}} t_{c}^{R}(p ", p, x ")
\end{aligned}
$$

with

$$
\begin{aligned}
& v_{c}^{R}\left(p^{\prime}, p, x^{\prime}, x\right) \equiv \int_{0}^{2 \pi} d \phi \\
& V_{c}^{R}\left(p^{\prime}, p, x^{\prime} x+\sqrt{1-x^{\prime 2}} \sqrt{1-x^{2}} \cos \phi\right) .
\end{aligned}
$$




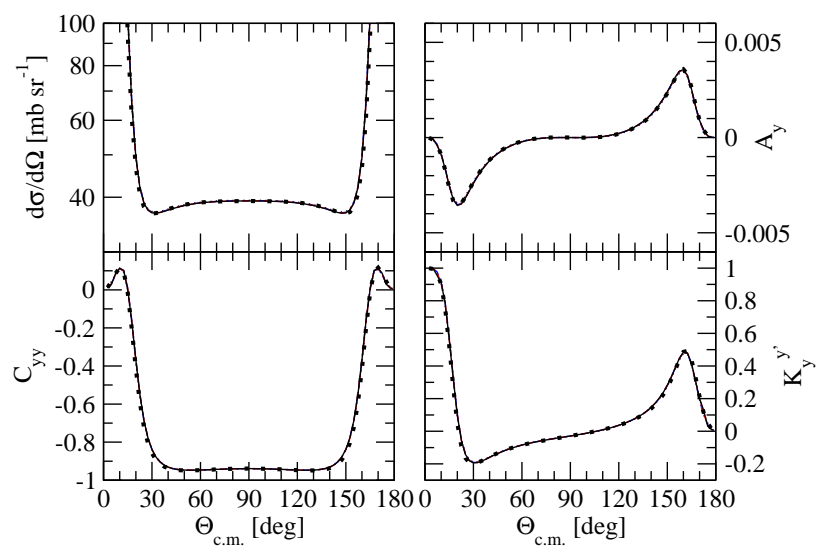

Fig. 2. (color online) The convergence of the pp scattering cross section $\left(\frac{d \sigma}{d \Omega}\right)$, analyzing power $\left(A_{y}\right)$, spin correlation coefficient $\left(C_{y y}\right)$, and spin transfer coefficient $\left(K_{y}^{y^{\prime}}\right)$ at $E_{p}^{l a b}=13 \mathrm{MeV}$ as a function of the c.m. scattering angle calculated with the screened Coulomb force and the CD Bonn nucleon-nucleon potential [13], which is kept for the partial waves up to $j \leq 3$. The screening radius is $R=120 \mathrm{fm}$ and $n=1$ (dotted line), $n=2$ (dasheddotted line), $n=3$ (dashed line), and $n=4$ (solid line). The curves for $n=1$ to $n=4$ all overlap on the scale of the figure. The exact Vincent-Phatak result is given by thick dots.

We solve Eq. (8) by discretizing all variables, generating the Neumann series and applying Padè summation.

For screening parameter $n=1$ the leading term in (8) can be calculated analytically

$$
\begin{aligned}
v_{c}^{R}\left(p^{\prime}, p, x^{\prime}, x\right) & =\frac{e^{2}}{\pi}\left(\left(p^{\prime 2}+p^{2}-2 p^{\prime} p x^{\prime} x+\frac{1}{R^{2}}\right)^{2}\right. \\
& \left.-4 p^{\prime 2} p^{2}\left(1-x^{\prime 2}\right)\left(1-x^{2}\right)\right)^{-\frac{1}{2}} .
\end{aligned}
$$

For $n>1$ this is no more possible and a two-dimensional numerical integration is required to get the leading term

$$
v_{c}^{R}\left(p^{\prime}, p, x^{\prime}, x\right)=\frac{e^{2}}{2 \pi^{2}} \int_{0}^{2 \pi} d \phi \int_{0}^{\infty} d r \frac{\sin (q r)}{q} e^{-\left(\frac{r}{R}\right)^{n}}
$$

where $q \equiv \sqrt{p^{2}+p^{\prime 2}-2 p p^{\prime}\left(x^{\prime} x+\sqrt{1-x^{\prime 2}} \sqrt{1-x^{2}} \cos \phi\right)}$. The detailed description, how we handle numerically this highly oscillating integral can be found in [7].

\section{The screened Coulomb t-matrix properties}

\subsection{The off-shell elements}

In this Subsection the three-dimensional t-matrix $t\left(p^{\prime}, p, x=\right.$ $\cos (\theta))$ will be shown as a two-dimensional function of momenta $p$ and $p^{\prime}$ at a given scattering angle $\theta$.

In Figs. 3 and 4 the real and imaginary parts of the t-matrix for $\mathrm{n}=4, \mathrm{R}=120 \mathrm{fm}$ and $\mathrm{E}=13 \mathrm{MeV}$ are shown at $\theta=45^{\circ}$ and $5^{\circ}$, respectively. These two values are chosen as representatives of intermediate and forward angles.
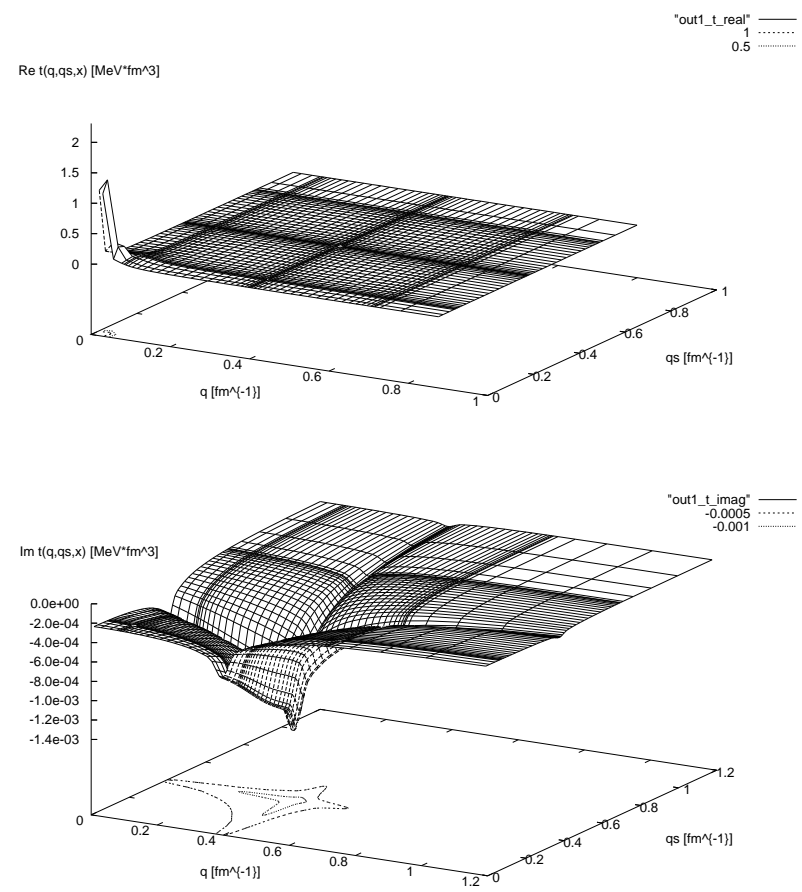

Fig. 3. The real (up) and imaginary (down) parts of the $t(q, q p, \cos (\theta))$ for the scattering angle $\theta=45^{\circ}$ at $\mathrm{E}=13 \mathrm{MeV}$. The screening parameters are $\mathrm{R}=120 \mathrm{fm}$ and $\mathrm{n}=4$.

The real part of $t$ has a steep maximum at small momenta at $\theta=45^{\circ}$, which evolves to a hump lying along diagonal $p^{\prime}=p$ for smaller angles. The spiky structure seen for the smallest angle comes only from the graphical representation on the finite grid of $p, p^{\prime}$-points. The increasing range of the hump shows that an action of the screened forces becomes more and more important for bigger momenta when moving to the smaller scattering angles. The imaginary part of the t-matrix has a minimum at the onshell point $p=p^{\prime}=k\left(\approx 0.396 \mathrm{fm}^{-1}\right.$ for $\left.\mathrm{E}=13 \mathrm{MeV}\right)$. Its absolute value is about three orders of magnitude smaller than the maximum of $\operatorname{Re}(t)$. The minimum of $\operatorname{Im}(t)$ becomes deeper and narrower with decreasing angle.

The pure off-shell Coulomb t-matrix is analytically given by $[2,10]$ :

$$
<\mathbf{p}^{\prime}\left|t_{c}^{R}\left(\frac{k^{2}}{m_{p}}\right)\right| \mathbf{p}>\rightarrow \frac{e^{2}}{2 \pi^{2}} \frac{1+I(x)}{\left(\mathbf{p}^{\prime}-\mathbf{p}\right)^{2}}
$$

where

$$
\begin{aligned}
& I(x)=\frac{1}{x} {\left[{ }_{2} F_{1}\left(1, i \eta ; 1+i \eta ; \frac{x+1}{x-1}\right)\right.} \\
&\left.-{ }_{2} F_{1}\left(1, i \eta ; 1+i \eta ; \frac{x-1}{x+1}\right)\right],
\end{aligned}
$$

$x^{2}=1+\frac{\left(p^{\prime 2}-k^{2}\right)\left(p^{2}-k^{2}\right)}{k^{2}\left(\mathbf{p}^{\prime}-\mathbf{p}\right)^{2}}$ and ${ }_{2} F_{1}$ is the hypergeometric function. Thus we are able to compare results of solving Eq.(8) with prediction (12) and check for which value of the screening radius the screening limit is achieved. This is presented in Fig. 5, where both parts of the off-shell screened 

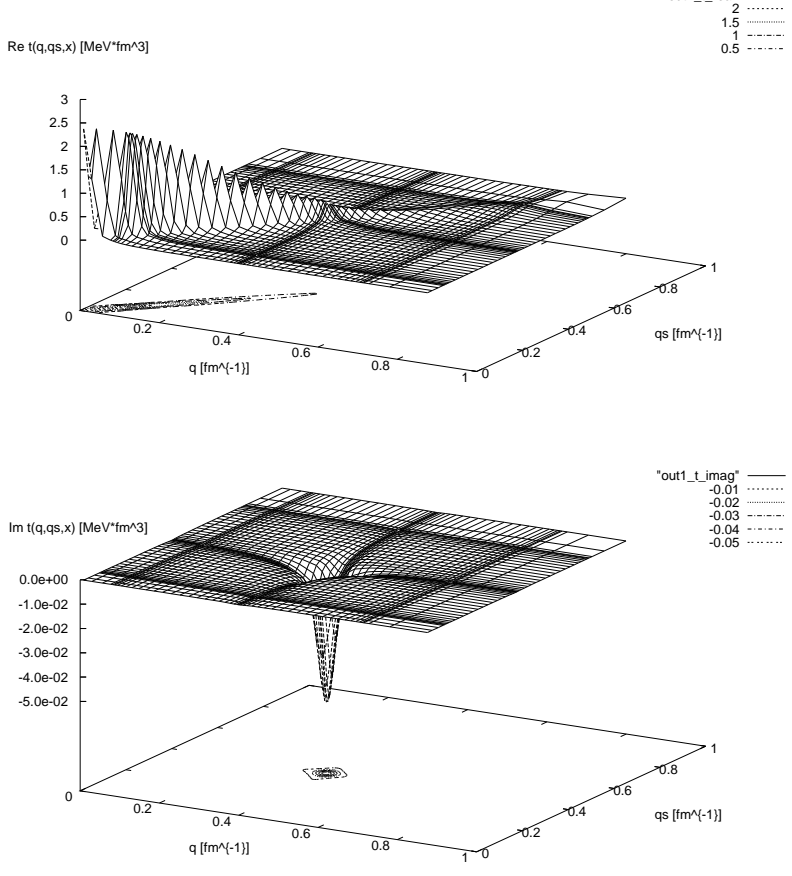

Fig. 4. The same as in Fig. 3 but for $\theta=5^{\circ}$.

Coulomb t-matrix $t_{c}^{R}\left(p, p^{\prime}, x\right)$ at $E_{p}^{l a b}=13 \mathrm{MeV}$ and fixed $p$ and $x$ values are shown as a function of $p^{\prime}$ and compared with pure Coulomb result. The small $R$-values $R=20 \mathrm{fm}$ and $R=60 \mathrm{fm}$ are insufficient, especially for the imaginary part, to reach the pure Coulomb off-shell values. However for bigger $R$-values, above $R \approx 100 \mathrm{fm}, t_{c}^{R}\left(p, p^{\prime}, x\right)$ approaches the screening limit. At this energy for $p^{\prime}=k=$ $0.396 \mathrm{fm}^{-1}$ one reaches the half-shell point where a discontinuity of $t$ matrix exists. In this ranges of $p^{\prime}$ bigger values of $R$ are needed.

\subsection{The half-shell elements}

Let us turn now to the half-shell pure Coulomb t-matrix, which is analytically given by [15].

$$
<\mathbf{p}^{\prime}\left|t_{c}^{R}\left(\frac{k^{2}}{m_{p}}\right)\right| \mathbf{k}>\rightarrow C_{0} e^{i \sigma_{0}} \frac{k \eta}{\pi^{2} q^{2}}\left(\frac{p^{2}-k^{2}}{q^{2}}\right)^{i \eta},
$$

where $\mathbf{q}=\mathbf{p}^{\prime}-\mathbf{k}$ is the momentum transfer, $\sigma_{0}=\Gamma(1+i \eta)$ is the pure Coulomb phase shift and $C_{0}^{2}=\frac{2 \pi \eta}{\exp ^{2 \pi \eta}-1}$ is the Coulomb penetrability. The direct comparison of this limit and the screened Coulomb half-shell t-matrix at $E_{p}^{l a b}=$ $13 \mathrm{MeV}$ is shown in Figs. 6 and 7 for the real and the imaginary part of $t$, respectively. On both figures, in upper row, discrepancy due to the oscillating factor $e^{i \Phi_{R}(k)}[3-5]$ is seen. The renormalization of $t$ matrix moves the screened half-shell t-matrix directly to its pure Coulomb limit (see lower row of Figs. 6 and 7). Especially the imaginary part of the half-shell screened t-matrix is affected by renormalization. The screening radii about $R=60 \mathrm{fm}$ are generally
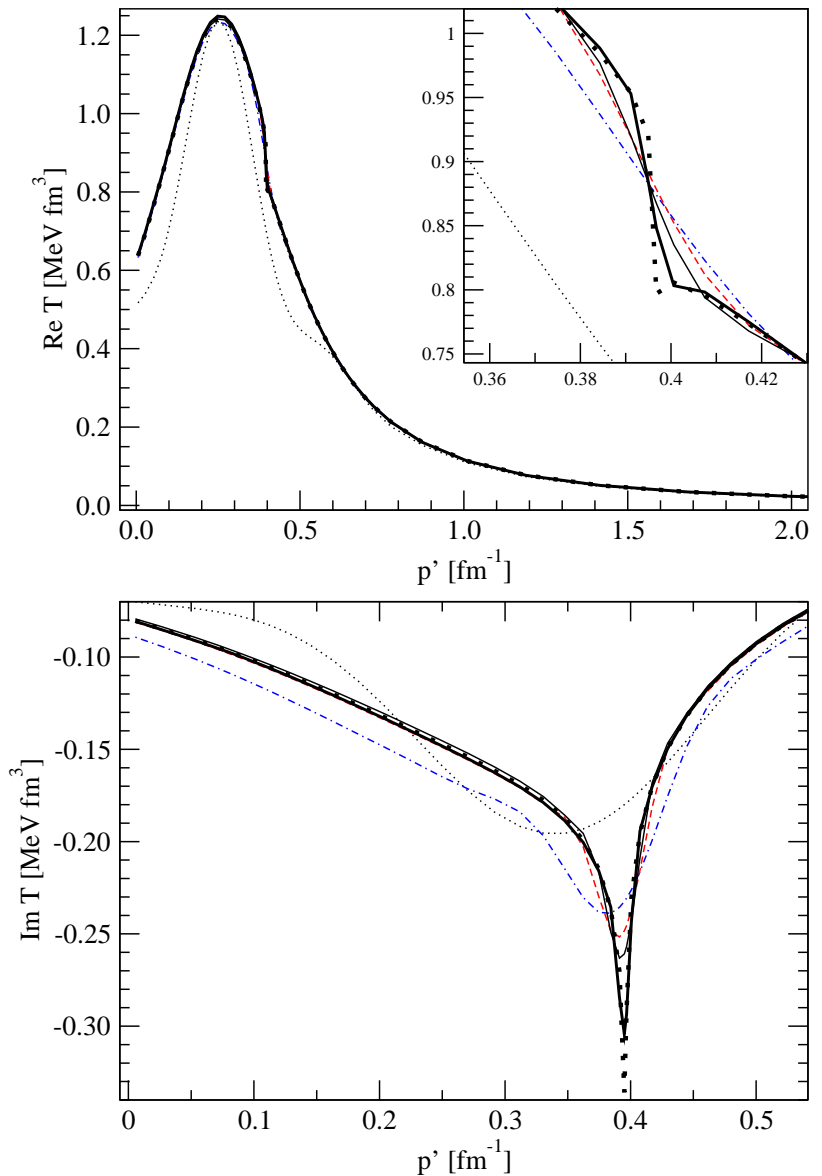

Fig. 5. (color online) Limiting behaviour of the real (upper) and the imaginary (lower) parts of the off-the-energy-shell screened tmatrix $t_{c}^{R}\left(p, p^{\prime}, x\right)$ at $E_{p}^{l a b}=13 \mathrm{MeV}, p=0.36 \mathrm{fm}^{-1}$, and $\theta=45^{\circ}$ as a function of the $p^{\prime}$ momentum for $n=4$ and different values of the screening radius $R: R=20 \mathrm{fm}$ (dotted line), $R=60 \mathrm{fm}$ (dashed-dotted line), $R=120 \mathrm{fm}$ (dashed line), $R=180 \mathrm{fm}$ (thin solid line), $R=500 \mathrm{fm}$ (thick solid line). The pure Coulomb off-shell result of Eq.(13) is given by thick dots. The half-shell situation is reached at $p^{\prime}=k=\sqrt{m_{p} E_{c . m .}}=0.396 \mathrm{fm}^{-1}$. In the inset a discontinuity develops if $p^{\prime}$ approaches $k$ from below or above.

sufficient to reach the pure Coulomb t-matrix. Although, for smaller angles one has to go to even bigger $R$ 's.

\subsection{The on-shell elements}

The on-shell elements of the screened Coulomb t-matrix, obtained from Eq. (8) at $E_{p}^{l a b}=13 \mathrm{MeV}$ are given in Figs. 8 (the real part) and 9 (the imaginary part). In the upper rows the results before renormalization are shown. In addition to them also the pure Coulomb amplitude is shown and represented by a thick solid line. A necessity for renormalization is clearly seen, especially for the imaginary part of $t$. For the on-shell elements the renormalization of $t$-matrix is done using the oscillating factor $e^{2 i \Phi_{R}(k)}$ [3-5]. Then the screened on-the-energy-shell t-matrix elements approach in the limit $R \rightarrow \infty$ the Coulomb scattering amplitude 
$19^{\text {th }}$ International IUPAP Conference on Few-Body Problems in Physics
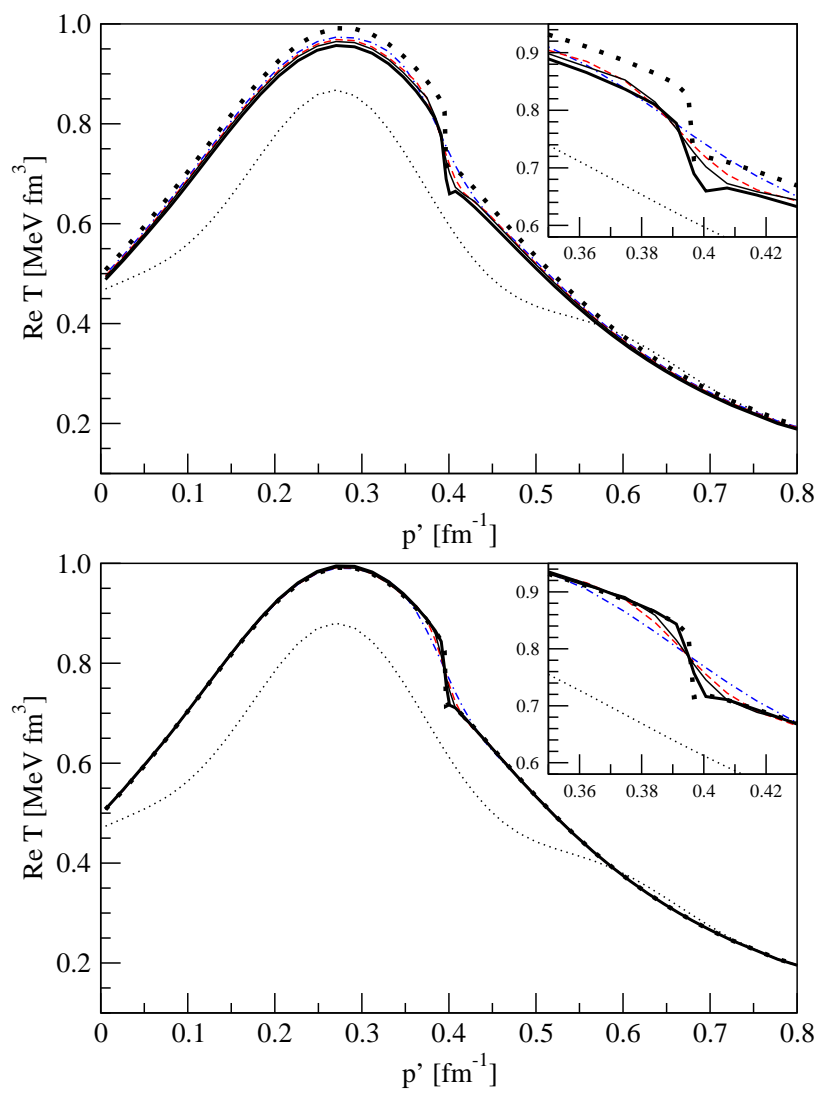

Fig. 6. (color online) The real part of the half-the-energy-shell screened t-matrix $t_{c}^{R}(k, p, x)$ before (upper row) and after (lower row) the renormalization. The proton lab. energy is $E=13 \mathrm{MeV}$, and $\theta=45^{\circ}$. The screening potential was taken with $n=4$ and different values of the screening radius $R: R=20 \mathrm{fm}$ (dotted line), $R=60 \mathrm{fm}$ (dashed-dotted line), $R=120 \mathrm{fm}$ (dashed line), $R=180 \mathrm{fm}$ (solid line) and $R=500 \mathrm{fm}$ (thick solid line) The pure Coulomb half-shell result of Eq.(14) is given by thick dots.

$A_{C}(\theta)[5,16]$

$$
\begin{aligned}
& \left.t_{c}^{R}(k, k, x)\right|_{\text {renormalized }} \equiv e^{-2 i \Phi_{R}(k)} t_{c}^{R}(k, k, x) \\
\rightarrow & -\frac{2}{m_{p}} \frac{A_{C}(\theta)}{(2 \pi)^{2}}=\frac{2}{m_{p}(2 \pi)^{2}} \frac{m_{p} e^{2}}{4 k^{2}} \frac{e^{-i \eta n\left(n \sin ^{2} \frac{\theta}{2}\right)}}{\sin ^{2} \frac{\theta}{2}} .
\end{aligned}
$$

Applying this procedure indeed shifts unrenormalized $t$-matrix to the pure Coulomb result. This is exemplified in the lower rows of Figs. 8 and 9. At this energy $\left(E_{p}^{l a b}=\right.$ $13 \mathrm{MeV}$ ) the screening range $R=120 \mathrm{fm}$ is sufficient to obtain screening limit at most scattering angles, only for very small ones the bigger values of $R$ are required.

\section{Summary}

We solved numerically the 3-dimensional LS equation for a screened Coulomb t-matrix with the exponential screening using different values of screening parameters. The resulting t-matrix taken on-shell together with a finite number of partial wave projected t-matrices generated by the
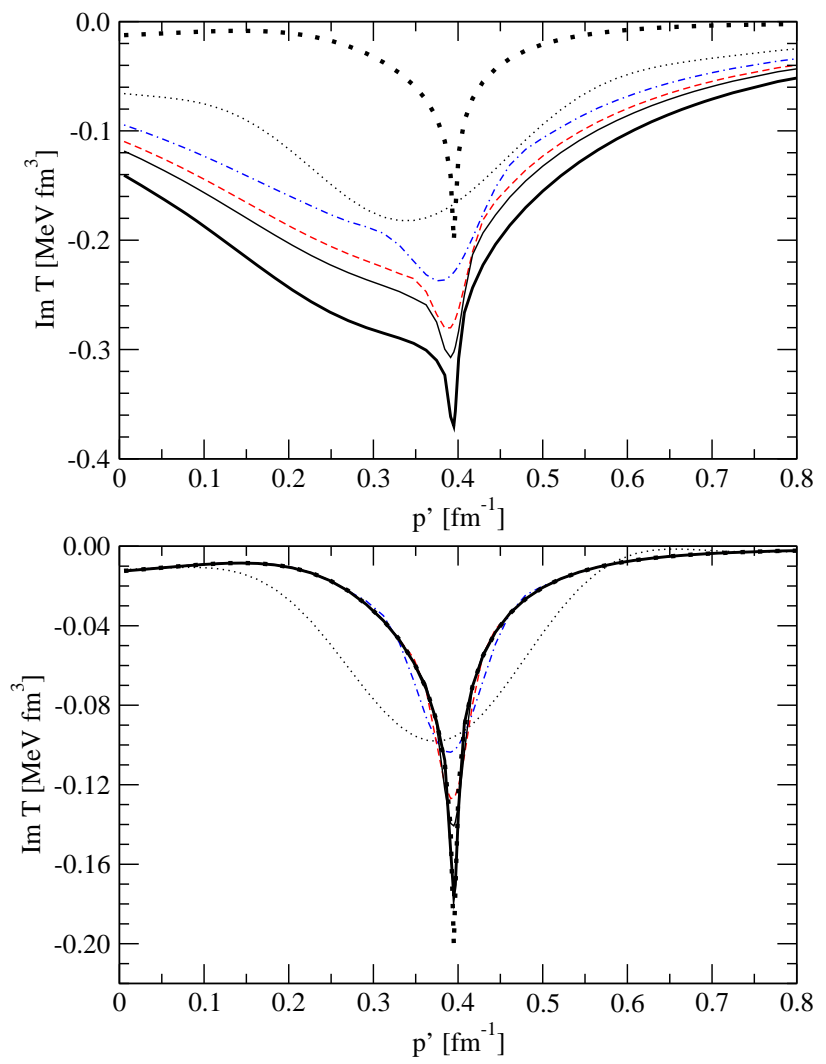

Fig. 7. (color online) The same as in Fig. 6 but for imaginary part of the half-the-energy-shell t-matrix.

screened Coulomb force and the nuclear force leads to correct $\mathrm{pp}$ observables. No renormalization is required in that case.

We also investigated numerically the screened 3-dimensional Coulomb t-matrix. The limits of the off-shell, halfshell and on-shell screened t-matrices were compared to the pure Coulomb values, which are known analytically. Our study confirms analytical results for the pure Coulomb force and allows to give the values of screening radii for which the screening limit is achieved. The resulting 3-dimensional screened Coulomb t-matrix is the important component of three-body calculations $[6,7]$ involving Coulomb interaction.

\section{Acknowledgments}

This work was supported by the Polish 2008-2011 science funds as the research project No. N N202 077435. It was also partially supported by the Helmholtz Association through funds provided to the virtual institute "Spin and strong QCD"(VH-VI-231) and by the European Community-Research Infrastructure Integrating Activity "Study of Strongly Interacting Matter" (acronym HadronPhysics2, Grant Agreement n. 227431) under the Seventh Framework Programme of EU. The numerical calculations have been performed on the supercomputer cluster of the JSC, Jülich, Germany. 


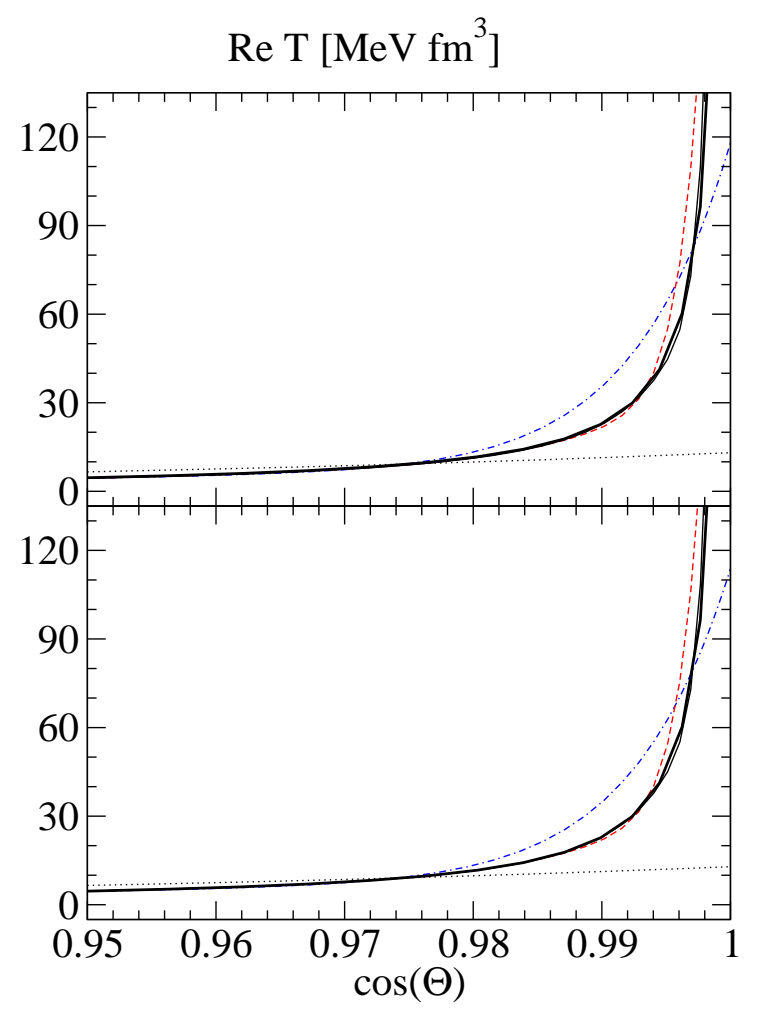

Fig. 8. (color online) The real part of the on-the-energy-shell screened t-matrix $t_{c}^{R}(k, k, x)$ (upper row) and the corresponding renormalized t-matrices (lower row) at $E_{p}^{l a b}=13 \mathrm{MeV}$ as a function of $x$ for $n=4$ and different values of the screening radius $R: R=20 \mathrm{fm}$ (dotted line), $R=60 \mathrm{fm}$ (dashed-dotted line), $R=120 \mathrm{fm}$ (dashed line), $R=180 \mathrm{fm}$ (thin solid line). The Coulomb on-shell amplitude of Eq.(15) is given by thick solid line. For other values of $\cos (\theta)$ all curves practically overlap.

\section{References}

1. R.Skibiński, J.Golak, H.Witała, W.Glöckle, Eur. Phys. J. A40, (2009) 215

2. J.C.Y. Chen and A.C. Chen, in Advances of Atomic and Molecular Physics, edited by D. R. Bates and J. Estermann ( Academic, New York, 1972), Vol. 8

3. J.R. Taylor, Nov's Cimento B23, (1974) 313

4. M.D. Semon and J.R. Taylor, Nuovo Cimento A26, (1975) 48

5. E. O. Alt, W. Sandhas, and H. Ziegelmann, Phys. Rev. C 17, (1978) 1981

6. H. Witała, J. Golak, R. Skibiński, and W.Glöckle, Eur. Phys. J. A41, (2009) 369

7. H. Witała, J. Golak, R. Skibiński, and W.Glöckle, Eur. Phys. J. A41, (2009) 385

8. W.F. Ford, Phys. Rev. 133, (1964) B1616

9. W.F. Ford, J. Math. Phys. 7, (1966) 626

10. L.P. Kok and H. van Haeringen, Phys. Rev. C 21, (1980) 512

11. M. Yamaguchi, H. Kamada, and Y. Koike, Prog. Theor. Phys. 114, (2005) 1323

12. C. M. Vincent and S. C. Phatak, Phys. Rev. C10, (1974) 391

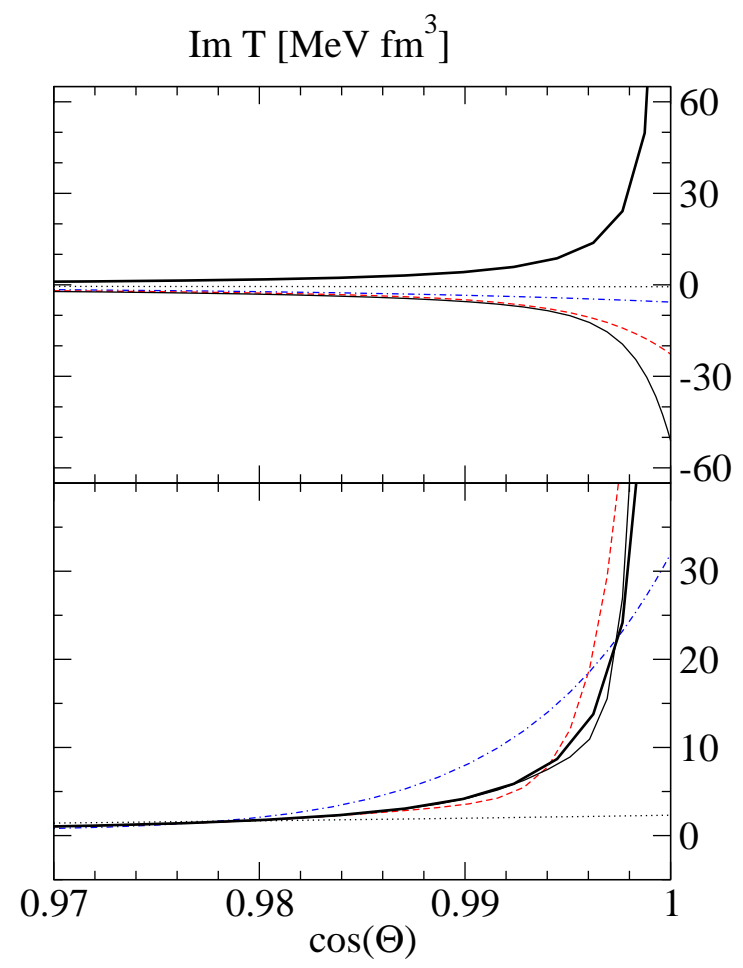

Fig. 9. (color online) The same as in Fig 8 but for the imaginary part o $t$.

13. R. Machleidt, F. Sammarruca, and Y. Song, Phys. Rev. C53, (1996) R1483

14. Ch. Elster, J.H. Thomas, and W. Glöckle, Few-Body Systems 24, (1998) 55

15. L.P. Kok and H. van Haeringen, Phys. Rev. Lett. 46, (1981) 1257

16. H. van Haeringen, Charged Particle Interactions, Theory and Formulas, Coulomb Press Leyden, 1985. 